\title{
Multi-Mode Resource-Constrained Project- Scheduling Problem With Renewable Resources: New Solution Approaches
}

Selcuk Colak, Cukurova University, Turkey

Anurag Agarwal, University of South Florida, USA

Selcuk Erenguc, University of Florida, USA

\begin{abstract}
We consider the multi-mode resource-constrained project scheduling problem (MRCPSP) with renewable resources. In MRCPSP, an activity can be executed in one of many possible modes; each mode having different resource requirements and accordingly different activity durations. We assume that all resources are renewable from period to period, such as labor and machines. A solution to this problem basically involves two decisions - (i) The start time for each activity and (ii) the mode for each activity. Given the NP-Hard nature of the problem, heuristics and metaheuristics are used to solve larger instances of this problem. A heuristic for this type of problem involves a combination of two priority rules - one for each of the two decisions. Heuristics generally tend to be greedy in nature. In this study we propose two non-greedy heuristics for mode selection which perform better than greedy heuristics. In addition, we study the effect of double justification and backward/forward scheduling for the MRCPS. We also study the effect of serial vs. parallel scheduling. We found that all these elements improved the solution quality. Finally we propose an adaptive metaheuristic procedure based on neural networks which further improves the solution quality. The effectiveness of these proposed approaches, compared to existing approaches in the literature, is demonstrated through empirical testing on two wellknown sets of benchmark problems.
\end{abstract}

Keywords: Project Management; Scheduling; Heuristics; Metaheuristics; Multi-Mode Resource-Constrained Project-Scheduling

\section{INTRODUCTION}

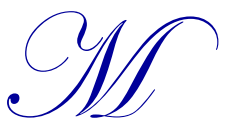

anaging projects efficiently and effectively is critical to organizational success in today's highly competitive business environment. We study the multi-mode resource-constrained projectscheduling problem (MRCPSP) which is a generalization of the classical resource-constrained project-scheduling problem (RCPSP). In RCPSP, activities of a project follow some precedence constraints and are processed in some predetermined duration of time using some predetermined amounts of resources. Resources are assumed to be limited for each period. The activities can be scheduled as they become resource and precedence feasible. The objective is to minimize the project completion time or makespan. The generalization of RCPSP occurs on two dimensions - first, in MRCPSP, each activity can be processed in multiple modes. A mode implies a certain level of resources used. The activity durations vary with the levels of resources used. Allocating more (or better quality) resources can accelerate activity duration. For example, an activity which takes two days to complete with three workers (mode 1) may be completed in only one day with six workers of the same skill level (mode 2) or with four workers of a higher skill level (mode 3). The second generalization has to do with the nature of resources. In MRCPSP, three types of resources are considered - renewable, non-renewable and doubly constrained (Slowinski, 1980). Renewable resources are available in limited quantities for each time period and are renewable from period to period (e.g. labor, machines). Non-renewable resources are limited for the entire project (e.g. project budget). Doubly-constrained resources are limited both for the entire project as well as for each period. 
The first generalization, the one that allows use of different levels of resources to be allocated to activities, makes the MRCPSP of much greater practical significance to the project manager than the single-mode version of the problem, the RCPSP. This is because in the real world, managers generally enjoy the prerogative of manipulating resource levels in order to accelerate critical activities to reduce the overall project duration. Yet, the MRCPSP is not as widely studied as the RCPSP. As far as the second generalization, which takes into account renewable, non-renewable and doubly-constrained resources, the renewable resource problem is most commonly found in practice because most resources are procured in markets through funding. Of course, if funding is limited, then all resources become non-renewable.

To the best of our knowledge, while the most general MRCPSP problem has received wide attention, the problem of only renewable resources has received very little attention in the literature. The only studies, to the best of our knowledge, on MRCPSP with only renewable resources are by Boctor (1993, 1996a and 1996b), Mori and Tseng (1997) and Alcaraz et al. (2003). Further, of the 19 sets of benchmark MRCPSP instances in PSPLIB, only one set is devoted to only renewable resources. Boctor (1993) has also developed a set of benchmark problems for the MRCPSP problems with renewable-only resources. Given the practical significance of this problem, there is a need to explore better solution approaches. In this paper, we develop new solution approaches for solving the MRCPSP with renewable resources only, hereafter MRCPSP-RR. The objective is to determine the execution mode and the start time of each activity in order to minimize the project completion time or the makespan, while satisfying the precedence and resource constraints. Activities are assumed to be non-preemptive, a common assumption in the literature. The MRCPSP-RR is a strongly NP-Hard problem as it is a generalization of the RCPSP which itself is strongly NP-Hard.

Formally, the MRCPSP-RR can be described as follows: A project consists of $N$ activities represented by index $i=1, \ldots, N$. The $N^{\text {th }}$ activity is the dummy terminal activity with no successors and a duration of zero. Each activity can be executed in one of $j$ modes, where $j$ goes from $l$ to $M_{i}$, where $M_{i}$ is the number of possible modes for activity $i$. Once a mode has been selected for an activity, it must be finished without switching the mode. The duration of activity $i$ executed in mode $j$ is $d_{i j}$. The non-preemptive assumption implies that once an activity $i$ has been assigned mode $j$, it must be executed for $d_{i j}$ consecutive time units without interruption. Activity $i$ cannot start before all of its predecessors have been completed. We assume there are $R_{k t}$ amount of renewable resources of type $k$ available in time period $t$. Index $k$ goes from $l$ to $K$, where $K$ is the total number of resource types. Activity $i$ executed in mode $j$ requires $r_{i j k}$ resource units per period for resource type $k$. The activity durations, resource availabilities and resource requirements per activity are non-negative integers. Further assume that $E_{i}$ is the earliest finish time and $L_{i}$ the latest finish time of activity $i . E_{i}$ is calculated using a forward pass of the PERT chart using the critical path method assigning the fastest mode to each activity. $L_{N}$ is then set equal to an upper bound $T$ where $T$ is the sum of activity durations of all activities with the slowest execution modes. $L_{i}$ for the rest of the activities are calculated using a backward pass of the PERT chart assuming the slowest resource mode is assigned to activities. Also let $P_{i}$ be the set of all preceding activities of activity $i$. We also define $x_{i j t}$ as a $0-1$ binary variable which assumes a value of 1 if activity $i$ is assigned to mode $j$ having a finish time of $t$ and zero otherwise. The integer programming formulation of the problem is described as:

Minimize: $\sum_{t=E_{N}}^{L_{N}} t . x_{N . t}$

Subject to:

$\sum_{j=1}^{M_{i}} \sum_{t=E_{i}}^{L_{i}} x_{i j t}=1 \quad i=1, \ldots, N$
$\sum_{j=1}^{M_{p}} \sum_{t=E_{p}}^{L_{p}} t \cdot x_{i j t} \leq \sum_{j=1}^{M_{i}} \sum_{t=E_{i}}^{L_{i}}\left(t-d_{i j}\right) . x_{i j t} \quad i=1, \ldots, N, p \in P_{i}$ 


$$
\sum_{i=1}^{N} \sum_{j=1}^{M_{i}} \sum_{s=t}^{t+d_{i j}-1} r_{i j k} \cdot x_{i j s} \leq R_{k t} \quad k=1, \ldots, K, t=1, \ldots, T
$$

Constraint set (1) ensures that each activity is executed only once, set (2) ensures that the precedence constraints are satisfied and set (3) ensures that the resource constraints are satisfied. For large $N$ the problem is difficult to solve optimally and therefore heuristics are used.

In solving this problem heuristically, two decisions are involved - (i) which activity to be assigned if two or more activities are precedence and resource feasible and (ii) which of the several feasible modes of execution to select for the assigned activity. Two priority rules are therefore needed to solve the problem - one for activity selection and one for execution-mode selection. These two priority rules working in tandem constitute a heuristic for solving this type of problem. If we considered $x$ number of rules for activity selection, and $y$ for mode selection, then the total number of rule combinations (heuristics) for the MRCPSP with renewable resources would be $x * y$. In this paper, we (i) two non-greedy heuristics (priority rules) for mode selection and a new greedy rule for activity selection, (ii) study the effect of double justification, (iii) study the effect of forward/backward scheduling, (iv) study the effect of serial vs. parallel scheduling and (v) propose an adaptive metaheuristic, based on neural network principles, to solve the problem iteratively using weighted activity-durations. While the effect of double justification and serial vs. parallel scheduling have been studied for the RCPSP, they have not been studied for the MRCPSP.

In the next section (Section 2), we review the relevant literature for the general MRCPSP as well as the MRCPSP with only renewable resources. Section 3 describes the existing heuristics in the literature and also the new heuristics developed in this study. The proposed adaptive metaheuristic is detailed in Section 4. In Section 5 we present the results of our computational experiments and compare our results with those of previous approaches in the literature. Summary and concluding remarks appear in Section 6.

\section{RELEVANT LITERATURE}

The MRCPSP was first introduced by Elmaghraby (1977). Talbot (1982) was the first to propose an exact solution method using the implicit enumeration scheme. Patterson et al. $(1989,1990)$ presented a more powerful backtracking procedure for solving the problem. These approaches, however, were unable to solve instances with more than 15 activities in reasonable computation time. Demeulemeester and Herroelen (1992) had proposed a branch-and-bound procedure for the RCPSP, which was extended to the MRCPSP by Sprecher et al. (1997). Hartman and Drexl (1998) compared the existing branch-and-bound algorithms and presented an alternative exact approach based on the concepts of mode and extension alternatives. Sprecher and Drexl (1998) proposed an exact solution procedure by extending the precedence-tree guided enumeration scheme of Patterson et al. $(1989,1990)$. Although their approach was the most powerful procedure, it was unable to solve the highly resource-constrained problems (more than 20 activities and two modes per activity) in reasonable computational times.

Several heuristics and metaheuristics have also been proposed in the literature for this problem. Drexl and Grunewald (1993) presented a stochastic scheduling method. Ozdamar and Ulusoy (1994) proposed a local constraint based approach that selects the activities and their respective modes locally at every decision point. Kolisch and Drexl (1997) developed a local search method that first finds a feasible solution and then performs a neighborhood search on the set of feasible mode assignments. Different genetic algorithms for solving the MRCPSP have been developed by Mori and Tseng (1997), Ozdamar (1999), Hartmann (2001) and Alcaraz et al. (2003). Simulated annealing algorithms are one of the most common heuristic procedures applied to MRCPSP. Slowinski et al. (1994), Bouleimen and Lecocq (2003) and Jozefowska et al. (2001) have suggested simulated annealing algorithms. Nonobe and Ibaraki (2002) proposed a tabu-search procedure for this problem. More recently, Lova et al, (2009) have proposed a hybrid genetic algorithm and Barrios et al. (2011) have proposed a Double genetic algorithm for this problem.

As far as MRCPSP_RR, Boctor (1993) was the first to consider that problem. He proposed and developed seven rules for prioritizing activities and three for prioritizing modes for a total of 21 different rule combinations. 
Details of these rules are presented in the next section. In a follow-up study, Boctor (1996a) proposed backward/forward scheduling to improve the results and also proposed a new priority rule for mode selection. The new rule, although very complex, gave better results. Boctor (1996b) proposed a simulated annealing approach and Alcaraz et al. (2003) proposed a genetic algorithm approach for this problem. Both simulated annealing and genetic algorithms improved the results from single-pass heuristics significantly.

In the next section, we will briefly describe the heuristics that have been used to solve MRCPSP problem and explain our proposed greedy and non-greedy heuristics.

\section{HEURISTIC METHODS FOR MRCPSP-RR}

\subsection{Existing Heuristics}

In the existing literature, parallel schedule generation scheme is used. In this scheme, a clock is maintained which is moved to a point when the next activity or activities become precedence-feasible and resource-feasible. Assignment takes place from amongst the activities that are ready for assignment, based on an activity priority rule. Note that for each precedence-feasible activity, not all execution modes may be feasible at a given time. Once an activity is selected, the mode-selection priority rule is applied to choose one of several feasible execution modes. Boctor (1993) used seven rules for prioritizing activities and three for prioritizing execution modes resulting in twenty one heuristics. The seven rules for prioritizing activities were minimum total slack (Min-SLK), minimum latest finish time (Min-LFT), maximum number of immediate successors (Max-NIS), maximum remaining work (Max-RWK), maximum processing time (Max-PTM), minimum processing time (Min-PTM) and maximum number of subsequent candidates (Max-CAN). Of these seven, Min-SLK, Max-RWK, Max-CAN and Min-LFT performed better than the other three. The three rules for prioritizing execution modes were: shortest feasible mode (SFM), least criticality ratio (LCR) and least resource proportion (LRP). Of these three, the SFM rule dominated the other two. So, the four best heuristics (or rule combinations) were Min-SLK/SFM, Max-RWK/SFM, Max-CAN/SFM and Min-LFT/SFM. Boctor, in a follow-up study (Boctor, 1996a), focused on these four heuristics and improved the results by solving twice: once forward and once backward and using the better of the two solutions. He also proposed a new rule for execution-mode selection, which we will call Boct96Rule, which gave better results than the SFM heuristic. This new rule attempted to prioritize execution modes based on certain conditions of non-dominated activity-mode combinations. Although complex to implement, this rule gave good results.

\subsection{Proposed Heuristics}

We propose several new heuristics for prioritizing activities and also for prioritizing execution modes. The rules for execution modes are non-greedy in nature and are designed to take into account the criticality of an activity when deciding on the execution mode. In addition, we propose the use of serial schedule generation scheme (SSGS) which has not been used for this problem. The seven activity priority rules by Boctor (1993) all employ parallel schedule generation scheme. In SSGS, the priority order of the activities is predetermined using a priority rule; assignment of activities occurs in that order at the first possible resource and precedence feasible opportunity. For details of SSGS see Kolisch (1996). To improve the solution quality, we also employ forward and backward scheduling as proposed by Boctor (1996a). In backward scheduling the precedence relationships of all activities are reversed. In other words, the direction of arrows on the PERT chart is reversed for each arrow and scheduling performed normally, treating the problem as a forward scheduling problem. Using the backward scheduling approach, for the same heuristic, a different schedule is generated with a different makespan. Probabilistically, therefore, fifty percent of the time, the backward schedule gives better solution than the forward schedule. We consider the better of forward and backward schedules. We also apply backward-forward improvement (BFI), also known in the literature as double justification. BFI has not been applied to the MRCPSP or the MRCPSP-RR problem in the literature. In BFI, once the forward schedule is generated using any heuristic, a backward schedule is generated using the activity priority rule of highest finish time using the actual finish times obtained during the forward schedule. This is followed by another pass of forward scheduling using the rule of lowest start times for activities using the start times obtained during the backward pass. The BFI procedure is an attempt to reduce the makespan, without altering the priority order of the activities. It basically tries to remove any unnecessary empty spaces in the Gantt chart. Valls et al. (2005) applied the BFI procedure for the RCPSP problem. 
As far as the rules for prioritizing activities, we use the best of the seven rules proposed by Boctor (1993), namely Max-RWK. We propose a new heuristic called minimum latest start time (Min-LST). This rule has been used in many other types of scheduling problems, so it is not new to the scheduling literature, but is new for MRCPSP-RR and it gave better results than the other seven activity priority rules used in Boctor (1996a).

For prioritizing execution modes we propose two new, non-greedy approaches which take into account the criticality of an activity. The non-greedy approaches differ from existing greedy approaches in that, a precedencefeasible activity may not be scheduled in spite of being resource feasible for some execution mode. In the first nongreedy rule, if the feasible execution mode is not the fastest execution mode, we take into account the time the activity would have to wait for the resources for the fastest mode and the difference between the activity durations between the fastest mode and the fastest feasible mode. Clearly, if it has to wait less than the difference between the activity durations, it makes sense to wait. The waiting strategy is what makes the rule non-greedy.

In the second non-greedy rule, a precedence-feasible activity considers all modes faster than the fastest feasible mode and checks against each one the difference between the activity durations and the amount of waiting. If the fastest mode is not worth waiting for then perhaps the next fastest mode might be worth waiting. The first non-greedy rule, discussed earlier, would not wait for the second fastest mode but the second rule would.

We call the first non-greedy rule for prioritizing execution mode the "Shortest Feasible Mode with Conditional Wait for the Fastest Mode" or SFM-CWFM, and the second rule "Shortest Feasible Mode with Conditional Wait for a Better Mode" or SFM-CWBM. We recommend taking the best of three mode selection rules - SFM, SFM-CWFM and SFM-CWBM. Figure 1 shows the pseudo-code of the proposed heuristic using SFMCWFM rule and Figure 2 shows the same with the SFM-CWBM rule.

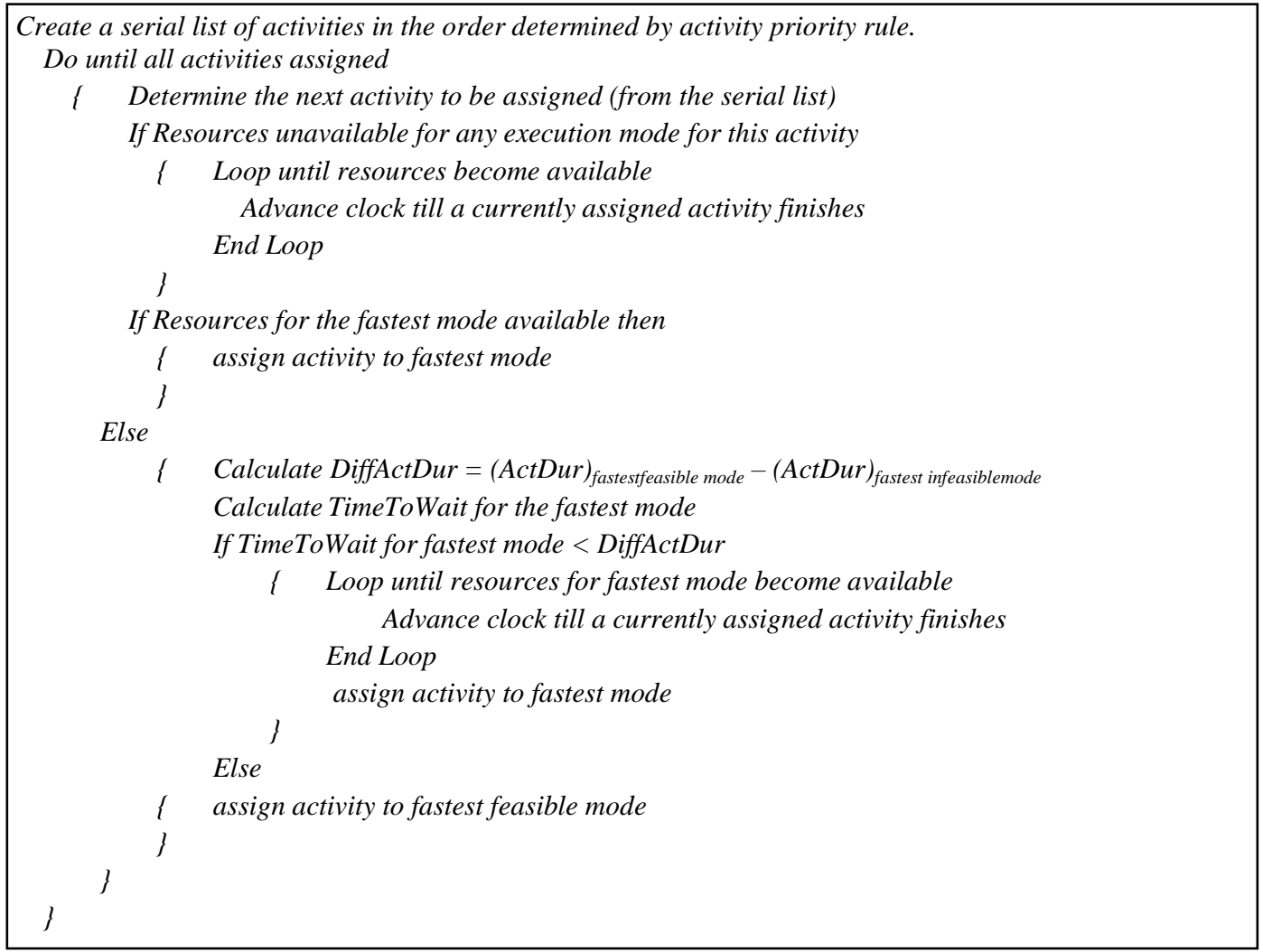

Figure 1: Algorithm for the SFM-CWFM Rule 


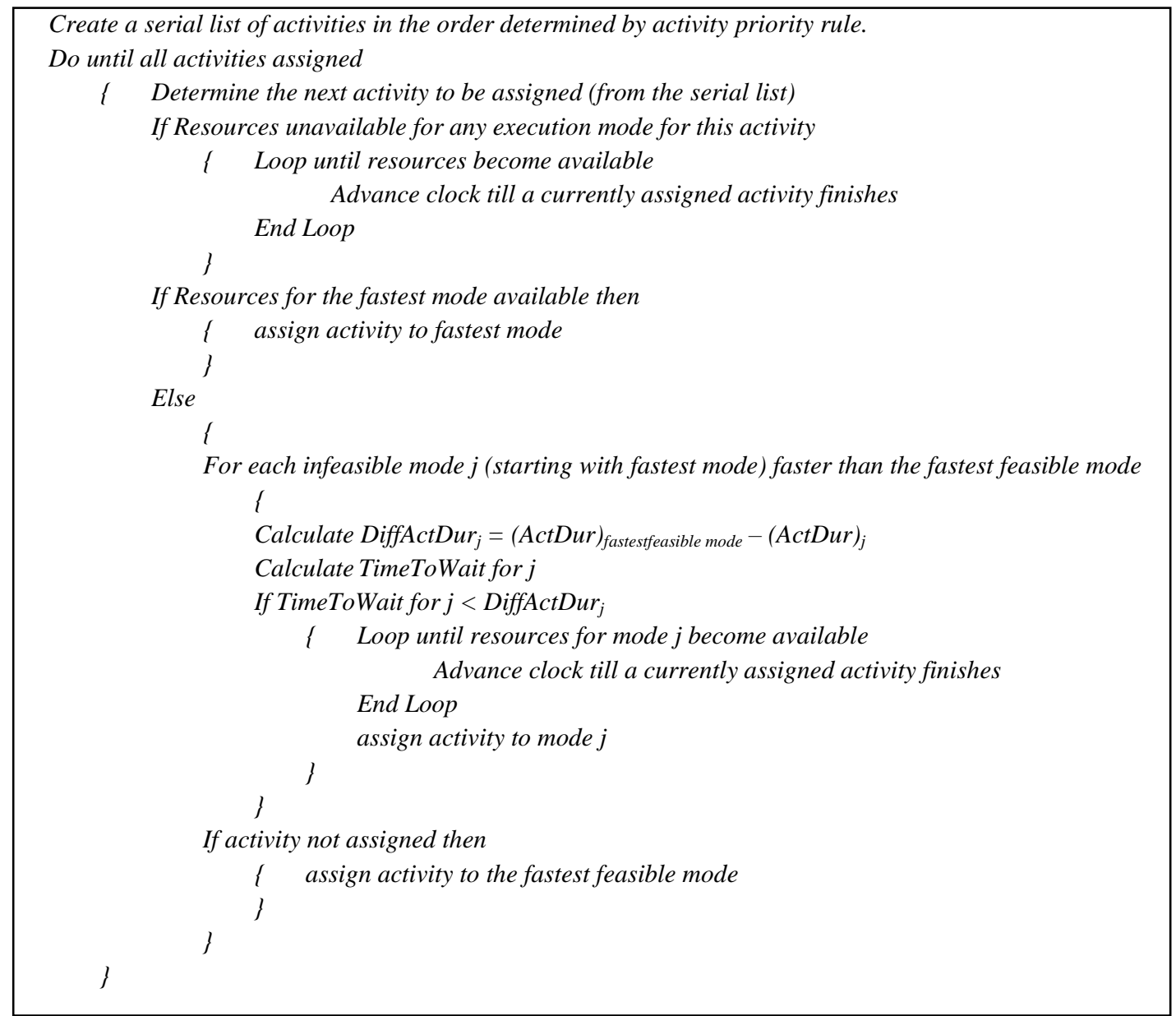

Figure 2: Algorithm for the SFM-CWBM Rule

We call this proposed approach ACE-SP (Agarwal, Colak and Erenguc - Single Pass). It consists of serial schedule generation scheme, better of backward and forward schedule and BFI or double justification and best of three mode selection rules. ACE-SP-LST is basically ACE-SP with Min-LST activity priority rule and ACE-SPRWK is ACE-SP with Max-RWK activity priority rule. The next section describes the adaptive metaheuristic we use.

\section{ADAPTIVE METAHEURISTIC}

Metaheuristics provide a good way to improve upon a heuristic solution iteratively in reasonable amount of computing time. We propose an adaptive metaheuristic in conjunction with each of the two heuristics (ACE-SPLST and ACE-SP-RWK) to improve the solution quality. We call the proposed metaheuristic the ACE-AM approach. ACE-AM-LST is the metaheuristic with Min-LST while ACE-AM-RWK is with Max-RWK. In this approach, a weight factor is used with each of the activity durations. Using the weighted activity durations, one of the basic heuristics such as Max-RWK/SFM or Max-RWK/SFM-CWBM or ACE-SP-LST or ACE-SP-RWK is applied. For the first iteration, the weights for all the activities are the same, hence the first iteration solution is identical to the single-pass heuristic solution. In the subsequent iterations, the weights are modified and weighted processing times recalculated and the same heuristic is applied. This approach allows a non-deterministic local search in the neighborhood of the heuristic solution. The basic idea in this approach is similar to neural networks where perturbation in the weight vector is used as a means of non-deterministic local search. The weight vector is modified using a weight modification strategy to find improved neighborhood solution in subsequent iterations. Using this adaptive search approach, the gaps are reduced significantly. This approach was first applied in Agarwal et al. (2006) for the flow-shop scheduling problem. 
The adaptive metaheuristic is explained next, using the following notation:

$k \quad$ Iteration counter

$k_{\max } \quad$ Max number of iterations

$d_{i j} \quad$ Activity duration of activity $i$ executed in mode $j$.

$w_{i} \quad$ Weight associated with the activity $i$.

$w d_{i j} \quad$ Weighted activity duration of activity $i$ executed in mode $j$.

$L S T_{i} \quad$ Latest start time of activity $i$.

$R W K_{i} \quad$ Maximum remaining work for activity $i$.

$M S_{k} \quad$ Makespan in the current iteration

$M S_{B} \quad$ Best makespan

$\alpha \quad$ Search coefficient

$W_{B} \quad$ Best weights

Step 1: Initialization

a. Initialize $w_{i}=1, i=1, \ldots, N$

b. Initialize the iteration counter $k$ to 1 .

Step 2: Calculate weighted processing times

Step 3: Determine priority list

a. Calculate $w d_{i j}=w_{i} * d_{i j}, i=1, \ldots, N$

a. Determine the priority list for the activities using a priority rule such as Min-LST or Max-RWK, where LST and RWK are calculated using $w d_{i j}$ instead of $d_{i j}$.

Step 4: Determine makespan

a. Find a feasible schedule using serial schedule generation scheme, using the priority list. In other words, schedule each activity at its earliest possible time given precedence and resource constraints using one of the three mode selection priority rules (SFM, SFM-CWFM, SFM-CWBM). This schedule gives us the makespan $m s_{k}$.

Step 5: Apply search modification strategy and modify weights

a. If $M S_{k}$ is the best makespan so far, save the current weights as best weights $\left(W_{B}\right)$ and the makespan as the best makespan $\left(M S_{B}\right)$.

b. If $k=k_{\max }$, go to Step 7 .

c. If $k<k_{\max }$, modify the weights using the following strategy:

a. Generate a random number RND between 0 and 1 using uniform distribution.

i. If RND $>0.5$ then $\left(w_{i}\right)_{k+1}=\left(w_{i}\right)_{k}+\mathrm{RND} * \alpha *$ error

ii. If RND $<=0.5$ then $\left(w_{i}\right)_{k+1}=\left(w_{i}\right)_{k}-\mathrm{RND} * \alpha *$ error

iii. error is the difference between the current makespan $\left(M S_{k}\right)$ and the lower bound $(L B)$ for the problem in question.

Step 6: Next iteration

a. Increment $k$ by one and go to step 2 .

Step 7: Display Solution

a. $M S_{B}$ is the solution. The schedule is generated using the $W_{B}$.

The search coefficient $(\alpha)$ used in Step 5c determines the degree of weight change per iteration. A higher coefficient leads to a greater change and vice versa. One could therefore control the granularity of the search by varying $\alpha$. The search coefficient should neither be too low nor too high. A low $\alpha$ will slow down convergence and make it difficult to jump local minima, while a high $\alpha$ will render the search too erratic or volatile to afford convergence. With some empirical trial and error, we found that a rate of 0.005 worked well for all the problems.

\section{COMPUTATIONAL EXPERIMENTS AND RESULTS}

Two benchmark problem sets are available in the literature for the MRCPSP-RR. The first one was created by Boctor (1993) and the second one is part of the PSPLIB (Kolisch and Specher, 1997) problems. In Boctor's set, there are 240 problem instances. These problems have been used by researchers as benchmark to demonstrate the 
effectiveness of their proposed approaches. These problems were generated randomly and are divided into two main subsets - 120 instances of fifty-activities and 120 of one hundred-activities. In each subset, there are 40 instances each with respectively 1,2 and 4 resource types. The average number of immediate successors is 2 and the number of execution modes for each activity is uniformly generated from 1 to 4 .

The PSPLIB benchmark problems are mainly for the general MRCPSP. There are 19 sets of problems, each set having roughly 400 to 600 problems. One of the 19 sets of problems (set n0.mm) has instances with only renewable resources. There are 470 problems in this set. Results on these 470 problems using other approaches are not available explicitly in the literature.

Our heuristics were coded in Visual Basic .Net running on Windows-XP® operating system and implemented on a Pentium-4 PC.

\subsection{Results for Boctor's Benchmark Problems}

Results for the Boctor's benchmark problems are presented now. Table 1 shows the average percent deviation from the critical-path based lower bound for the six single-pass heuristic combinations Min-LST-SFM, Min-LST-SFM-CWFM, Min-LST-SFM-CWBM, Max-RWK-SFM, Max_RWK-SFM-CWFM and Max-RWKSFM-SWBM. The lower bound is calculated using the shortest mode for each activity and assuming infinite resources.

Table 1: Comparison of Proposed Single-Pass Greedy and Non-Greedy Heuristics Average Percentage Deviation from the Critical-Path Based Lower Bound

\begin{tabular}{lccccccccc}
\hline $\begin{array}{c}\text { Num } \\
\text { of } \\
\text { Activities }\end{array}$ & $\begin{array}{c}\text { Num of } \\
\text { Resource } \\
\text { Types }\end{array}$ & $\begin{array}{c}\text { Min- } \\
\text { LST- } \\
\text { SFM }\end{array}$ & $\begin{array}{c}\text { Min- } \\
\text { LST- } \\
\text { SFM- } \\
\text { CWFM }\end{array}$ & $\begin{array}{c}\text { Min- } \\
\text { LST- } \\
\text { SFM- } \\
\text { CWBM }\end{array}$ & $\begin{array}{c}\text { Min- } \\
\text { LST } \\
\text { Best of } \\
\text { Three }\end{array}$ & $\begin{array}{c}\text { Max- } \\
\text { RWK- } \\
\text { SFM }\end{array}$ & $\begin{array}{c}\text { Max- } \\
\text { RWK- } \\
\text { SFM- } \\
\text { CWFM }\end{array}$ & $\begin{array}{c}\text { Max- } \\
\text { RWK- } \\
\text { SFM- } \\
\text { CWBM }\end{array}$ & $\begin{array}{c}\text { Max- } \\
\text { RWK } \\
\text { Best of } \\
\text { Three }\end{array}$ \\
\hline 50 & 1 & 18.46 & 17.81 & 17.86 & 16.99 & 18.66 & 18.19 & 18.19 & 17.44 \\
50 & 2 & 33.50 & 33.85 & 33.68 & 32.32 & 33.59 & 33.94 & 33.75 & 32.36 \\
50 & 4 & 45.08 & 45.79 & 45.87 & 44.31 & 45.38 & 45.72 & 45.79 & 44.48 \\
100 & 1 & 18.31 & 17.25 & 17.27 & 16.91 & 18.15 & 17.38 & 17.35 & 16.95 \\
100 & 2 & 36.68 & 36.23 & 36.23 & 35.50 & 37.05 & 36.29 & 36.29 & 35.84 \\
100 & 4 & 47.38 & 46.39 & 46.39 & 45.96 & 47.02 & 46.07 & 46.04 & 45.65 \\
\hline All probs. & & 33.23 & 32.89 & 32.88 & 32.00 & 33.31 & 32.93 & 32.90 & 32.12 \\
\hline
\end{tabular}

Table 2 provides a comparison of these results with Boctor's results on the average percent deviation measure. When we take the best of six heuristics, the average gap for all problems is 31.4 percent compared to Boctor's best results of 34.4 percent. Even individually, the average gaps for the six heuristics range from 32.00 to 33.23 , all being better than the 34.4 percent.

Table 2: Comparison of Single-Pass Heuristics Average Percentage Deviation from the Critical-Path Based Lower Bound

\begin{tabular}{lccccccc}
\hline & $\begin{array}{c}\text { Boctor } \\
(\mathbf{1 9 9 6 a})\end{array}$ & $\begin{array}{c}\text { Boctor } \\
(\mathbf{1 9 9 6 a})\end{array}$ & $\begin{array}{c}\text { Boctor } \\
(\mathbf{1 9 9 6 a})\end{array}$ & ACE-SP & ACE-SP & ACE-SP \\
\hline $\begin{array}{c}\text { Num of } \\
\text { Activities }\end{array}$ & $\begin{array}{c}\text { Num of } \\
\text { Resource Types }\end{array}$ & $\begin{array}{c}\text { Min- } \\
\text { SLK-SFM }\end{array}$ & $\begin{array}{c}\text { Max- } \\
\text { RWK-SFM }\end{array}$ & $\begin{array}{c}\text { Boct96 } \\
\text { Rule }\end{array}$ & Min-LST & Max-RWK & $\begin{array}{c}\text { Best of } \\
\text { Two }\end{array}$ \\
\hline 50 & 1 & 21.9 & 22.8 & 20.2 & 17.0 & 17.4 & 16.7 \\
50 & 2 & 36.3 & 35.6 & 33.9 & 32.3 & 32.4 & 31.7 \\
50 & 4 & 49.2 & 48.1 & 46.4 & 44.3 & 44.5 & 43.6 \\
100 & 1 & 22.7 & 22.9 & 20.4 & 16.9 & 17.0 & 16.5 \\
100 & 2 & 40.3 & 40.5 & 37.4 & 35.5 & 35.8 & 35.0 \\
100 & 4 & 50.6 & 50.3 & 47.8 & 46.0 & 45.6 & 45.1 \\
\hline All probs. & & 36.8 & 36.7 & 34.4 & 32.0 & 32.1 & 31.4 \\
\hline
\end{tabular}


Table 3: Comparison of Single-Pass Heuristics Maximum Deviation from the Critical-Path Based Lower Bound

\begin{tabular}{lcccccc}
\hline & & $\begin{array}{c}\text { Boctor } \\
\text { (1996a) }\end{array}$ & $\begin{array}{c}\text { Boctor } \\
(\mathbf{1 9 9 6 a )}\end{array}$ & $\begin{array}{c}\text { Boctor } \\
(\mathbf{1 9 9 6 a})\end{array}$ & ACE-SP & ACE-SP \\
\cline { 3 - 7 } \begin{tabular}{c} 
Num of \\
\cline { 2 - 7 }
\end{tabular} & $\begin{array}{c}\text { Num of } \\
\text { Resource Types }\end{array}$ & $\begin{array}{c}\text { Min- } \\
\text { SLK-SFM }\end{array}$ & $\begin{array}{c}\text { Max- } \\
\text { RWK-SFM }\end{array}$ & $\begin{array}{c}\text { Boct96 } \\
\text { Rule }\end{array}$ & $\begin{array}{c}\text { Min- } \\
\text { LST }\end{array}$ & $\begin{array}{c}\text { Max- } \\
\text { RWK }\end{array}$ \\
\hline 50 & 1 & 35.9 & 41.8 & 30.4 & 28.0 & 29.1 \\
50 & 2 & 50.5 & 52.6 & 46.0 & 48.8 & 47.3 \\
50 & 4 & 67.9 & 59.8 & 60.3 & 57.8 & 57.4 \\
100 & 1 & 33.4 & 34.3 & 31.1 & 25.9 & 27.1 \\
100 & 2 & 55.0 & 54.3 & 48.5 & 52.4 & 53.3 \\
100 & 4 & 66.7 & 69.3 & 60.2 & 59.0 & 58.2 \\
\hline All probs. & & 67.9 & 69.3 & 60.3 & 59.0 & 58.2 \\
\hline
\end{tabular}

Table 4: Comparison of Single-Pass Heuristics Minimum Deviation from the Critical-Path Based Lower Bound

\begin{tabular}{lcccccc}
\hline & & $\begin{array}{c}\text { Boctor } \\
(\mathbf{1 9 9 6 a )}\end{array}$ & $\begin{array}{c}\text { Boctor } \\
(\mathbf{1 9 9 6 a )}\end{array}$ & $\begin{array}{c}\text { Boctor } \\
\mathbf{( 1 9 9 6 a )}\end{array}$ & ACE-SP & ACE-SP \\
\cline { 3 - 7 } $\begin{array}{c}\text { Num of } \\
\text { Activities }\end{array}$ & $\begin{array}{c}\text { Num of } \\
\text { Resource Types }\end{array}$ & $\begin{array}{c}\text { Min- } \\
\text { SLK-SFM }\end{array}$ & $\begin{array}{c}\text { Max- } \\
\text { RWK-SFM }\end{array}$ & $\begin{array}{c}\text { Boct96 } \\
\text { Rule }\end{array}$ & $\begin{array}{c}\text { Min- } \\
\text { LST }\end{array}$ & $\begin{array}{c}\text { Max- } \\
\text { RWK }\end{array}$ \\
\hline 50 & 1 & 10.9 & 8.9 & 11.3 & 5.7 & 7.2 \\
50 & 2 & 22.5 & 18.2 & 18.8 & 17.3 & 18.6 \\
50 & 4 & 35.6 & 35.6 & 32.8 & 32.3 & 31.9 \\
100 & 1 & 14.1 & 14.9 & 12.8 & 2.6 & 8.2 \\
100 & 2 & 28.0 & 27.4 & 27.4 & 37.4 & 23.5 \\
100 & 4 & 41.2 & 41.3 & 39.7 & 37.9 & 37.0 \\
\hline All probs. & & 10.9 & 8.9 & 11.3 & 5.7 & 7.2 \\
\hline
\end{tabular}

Tables 3 and 4 compare the ACE heuristics with Boctor's results on two other measures - maximum and minimum deviations from the lower bound. The maximum deviations for the two ACE-SP heuristics are 59\% and $58.2 \%$ compared to $60.3 \%$ obtained by Boctor96 rule. The minimum deviations are $5.7 \%$ and $7.2 \%$ compared to $11.3 \%$ of Boctor96 rule.

The results of the adaptive metaheuristic (ACE-AM) are now explained. For the adaptive metaheuristic, the search coefficient $\alpha$ was set to 0.005 and the weights were initialized at 1 . The determination of the best value of the search coefficient required some trial and error on a small set of problems. We report results for solutions obtained using 5,000 iterations. Average CPU times of our adaptive metaheuristic with Min-LST were 6.7 seconds for 5,000 iterations. The evaluation criterion we use is the average percentage deviation from the critical-path-based lower bound. Table 5 shows the results of ACE-AM for LST and RWK heuristics for 5,000 iterations. The results of this study are compared with those of the simulated-annealing approach of Boctor (1996b) and genetic algorithm approach of Alcaraz et al. (2003). For the entire set of 240 problems, the best of ACE-AM-LST and ACE-AMRWK gives a deviation of $25.8 \%$ from the lower bound while the same deviations using simulated annealing and genetic algorithms were $26.5 \%$ and $27.8 \%$, respectively. Even without considering the best of Min-LST and MaxRWK, each of the proposed approaches outperforms the existing approaches.

Table 5: Comparison of Metaheuristics Average Percentage Deviation from the Critical-Path Based Lower Bound

\begin{tabular}{lcccccc}
\hline & & $\begin{array}{c}\text { Boctor } \\
\mathbf{( 1 9 9 6 )}\end{array}$ & Alcaraz et al. (2003) & ACE-AM & ACE-AM & ACE-AM \\
\cline { 2 - 6 } Num of activities & $\begin{array}{c}\text { Num of } \\
\text { Resource types }\end{array}$ & Sim. Anneal. & $\begin{array}{c}\text { GA } \\
\mathbf{5 0 0 0} \text { Iter }\end{array}$ & $\begin{array}{c}\text { LST } \\
\mathbf{5 0 0 0} \text { Iter }\end{array}$ & $\begin{array}{c}\text { RWK } \\
\mathbf{5 0 0 0} \text { Iter }\end{array}$ & $\begin{array}{c}\mathbf{5 0 0 0} \\
\text { Iter }\end{array}$ \\
\hline 50 & 1 & 14.1 & NA & 13.1 & 13.2 & 12.8 \\
50 & 2 & 25.9 & NA & 25.5 & 25.5 & 25.1 \\
50 & 4 & 37.1 & NA & 36.8 & 36.9 & 36.7 \\
All 50 & 1 & 26.5 & 25.1 & 25.2 & 24.9 \\
100 & 2 & 14.2 & NA & 13.9 & 13.9 & 13.6 \\
100 & 4 & 29.4 & NA & 29.2 & 29.4 & 28.9 \\
100 & & 38.0 & NA & 38.1 & 38.2 & 37.8 \\
\hline All 100 & & 27.2 & 29.2 & 27.1 & 27.2 & 26.8 \\
\hline All problems & & 26.5 & 27.8 & 26.1 & 26.2 & 25.8 \\
\hline
\end{tabular}




\subsection{Results for PSPLIB Problems}

There are 470 problems in the n0.mm dataset of the PSPLIB benchmark problems for MRCPSP. Results for these 470 problems do not appear explicitly in the literature. Optimum solutions using exact approaches are known. The number of activities in these problems range from 12 to 22 . So they are not quite as large as Boctor's problems and are therefore solvable optimally using exact methods. We provide the gaps from the optimal solutions and also show how many of the 470 problems were solved to optimality. Table 6 shows the results of using Parallel and Serial SGS, with various combinations of forward/backward and BFI and Mode selection heuristics.

Table 6: Average Gap from Optimal and Number of Problems Solved to Optimality for PSPLIB n0.mm Dataset Using

\begin{tabular}{|c|c|c|c|c|c|c|c|}
\hline \multicolumn{8}{|c|}{ Various Procedures } \\
\hline SGS & $\begin{array}{c}\text { Fwd or } \\
\text { Fwd/Bwd }\end{array}$ & $\begin{array}{c}\text { BFI or } \\
\text { not }\end{array}$ & $\begin{array}{c}\text { Greedy or Non- } \\
\text { Greedy Mode } \\
\text { Selection Rule }\end{array}$ & $\begin{array}{l}\text { Single-Pass Or } \\
\text { Metaheuristic }\end{array}$ & $\begin{array}{c}\text { Number } \\
\text { Of } \\
\text { Iterations }\end{array}$ & $\begin{array}{l}\text { Average Gap } \\
\text { in Percent } \\
\text { from Optimal }\end{array}$ & $\begin{array}{c}\text { Number of } \\
\text { Optimal Solutions } \\
\text { Found (out of 470) }\end{array}$ \\
\hline Par & Fwd only & No BFI & Greedy & Single-LFT & 1 & 10.88 & 226 \\
\hline Par & Fwd only & No BFI & Non-Greedy & Single-LFT & 1 & 4.77 & 276 \\
\hline Par & Fwd-Bwd & No BFI & Greedy & Single-LFT & 1 & 6.91 & 263 \\
\hline Par & Fwd-Bwd & No BFI & Non-Greedy & Single-LFT & 1 & 3.31 & 306 \\
\hline Par & Fwd-Bwd & BFI & Greedy & Single-LFT & 1 & 6.28 & 275 \\
\hline Par & Fwd-Bwd & BFI & Non-Greedy & Single-LFT & 1 & 2.65 & 326 \\
\hline Par & Fwd-Bwd & BFI & Non-Greedy & Meta-LFT & 1000 & 1.82 & 350 \\
\hline Par & Fwd-Bwd & BFI & Non-Greedy & Meta-LFT & 5000 & 1.12 & 371 \\
\hline Ser & Fwd only & No BFI & Greedy & Single-LST & 1 & 11.35 & 233 \\
\hline Ser & Fwd only & No BFI & Non-Greedy & Single-LST & 1 & 5.12 & 280 \\
\hline Ser & Fwd-Bwd & No BFI & Greedy & Single-LST & 1 & 7.74 & 266 \\
\hline Ser & Fwd-Bwd & No BFI & Non-Greedy & Single-LST & 1 & 3.48 & 308 \\
\hline Ser & Fwd-Bwd & BFI & Greedy & Single-LST & 1 & 5.25 & 286 \\
\hline Ser & Fwd-Bwd & BFI & Non-Greedy & Single-LST & 1 & 2.77 & 326 \\
\hline Ser & Fwd-Bwd & BFI & Non-Greedy & Meta-LST & 1000 & 1.25 & 371 \\
\hline Ser & Fwd-Bwd & BFI & Non-Greedy & Meta-LST & 5000 & 0.69 & 404 \\
\hline
\end{tabular}

Although for single-pass, parallel SGS performed marginally better than serial SGS. For example, parallel SGS for single pass gave a 2.65 percent gap vs. a 2.77 percent gap by serial SGS. For the adaptive metaheuristic, Serial SGS gave much superior results. For 5000 iterations, serial SGS gave an average gap of only 0.69 percent compared to a gap of 1.12 percent for parallel SGS. Introduction of Non-Greedy rule for model selection resulted in significant improvement. For example, for parallel SGS, for forward only and no BFI procedure, the gap reduced from 10.88 percent to 4.77 percent. For serial SGS, the non-greedy procedure helped reduce the gap from 11.35 percent down to 5.12 percent. Use of Forward-Backward scheduling also resulted in significant improvements. For example, for parallel SGS, the gap reduced from 10.88 percent to 6.91 percent by applying forward-backward procedure. Use of BFI resulted in marginal improvements. For example, for parallel SGS, use of BFI reduced the gap from 6.91 percent down to 6.28 percent. Of course, the use of adaptive metaheuristic further improved the results. The improvement owing to metaheuristics is particularly remarkable with the Serial SGS (from 2.77 down to 0.69 percent) compared to Parallel SGS (from 2.65 percent to 1.12 percent). The average CPU time for the single-pass heuristics were 0.0002 seconds. For the metaheuristics, for 1000 iterations it was 0.181 seconds and for 5000 iterations it was 0.821 seconds.

\section{SUMMARY AND CONCLUSIONS}

In this paper, we have considered the multi-mode resource-constrained project-scheduling problem with only renewable resources. This problem, we argue is of greater importance to the project manager than the RCPSP in which only single mode of execution is considered. We proposed several new solution approaches which include (i) a new greedy rule for prioritizing activities, (ii) two non-greedy rules for prioritizing execution modes, (iii) an adaptive metaheuristic, (iv) serial schedule generation scheme, (v) backward-forward scheduling and (vi) double justification or backward-forward improvement. The new greedy rule for prioritizing activity is called the 'Minimum Latest Start Time' or Min-LST. The non-greedy rules for mode selection use a waiting strategy, in 
which, a precedence-feasible activity may not be scheduled in spite of being resource feasible for at least one execution mode and waits for the fastest mode or one of the faster modes if the wait is worth it. These rules are called shortest feasible mode with conditional wait for fastest mode (SFM-CWFM) and shortest feasible mode with conditional wait for better mode (SFM-CWBM). We also proposed an adaptive metaheuristic, in which we use weighted activity-durations instead of given activity durations. A single-pass heuristic is applied iteratively to the problem using weighted activity-durations. The weights, which are the same for all activities for the first iteration, are modified at the end of each iteration. We also studied the effect of applying serial schedule generation scheme and found that it gave better results than parallel schedule generation scheme. Backward forward scheduling and double justification schemes were also applied, each of which provided some improvement.

The proposed algorithms were tested on two sets of benchmark problems from the literature. The results demonstrated that for Boctor's benchmark problems, the non-greedy rules for mode selection were slightly better than their greedy counterparts, the proposed greedy rule for activity selection performed slightly better than previously proposed rules and the adaptive metaheuristic outperformed the simulated annealing algorithm of Boctor (1996b) and genetic algorithm of Alcaraz et al. (2003). The gaps between the obtained solution and the critical path based lower bound using the proposed approaches was about $10 \%$ lower than the competitive approaches in the literature. Each element of the proposed approach contributed a little towards the reduction in the gap. For the PSPLIB benchmark problems, our approach was within $1.5 \%$ of the optimal, with 374 problems solved to optimality using 1000 iterations.

From the managerial perspective, such significant reductions in the makespan can amount to huge cost savings for the project. In a competitive business environment in which project completion deadlines are strictly imposed and penalties for missing the deadlines can be high, generating a better schedule can help avert those penalties. In competitive bidding environment, organizations can bid lower to win contracts.

We encourage more future research in this area. To many project managers a criterion other than makespan minimization might be of more interest. To some, a multi-criteria objective may be important. Heuristics and metaheuristics for these different objectives need to be developed for this problem.

\section{AUTHOR INFORMATION}

Selcuk Colak is an Associate Professor in the Department of Business at the Cukurova University, Adana, Turkey. His current research interests are: heuristics and metaheuristics, genetic algorithms, neural networks, project and machine scheduling, distribution planning. Professor Colak teaches Operations Management, Project Management and Logistics Management courses both undergraduate and graduate level. Selcuk Colak received his B.S. in Electrical and Electronics Engineering in 1997 from the Cukurova University in Adana, Turkey. He received his M.S. in Electrical and Computer Engineering in 2000 and his Ph.D. in Information Systems and Operations Management in 2006 from the University of Florida, Gainesvlle, FL, USA. E-mail: scolak@cu.edu.tu

Dr. Anurag Agarwal is a Professor in the Department of Information Systems and Decision Sciences at University of South Florida, Sarasota, FL, USA. He earned his Ph.D. from The Ohio State University, Columbus, OH, USA (1993) and an MBA from the University of Wisconsin, La Crosse, WI, USA (1988). He teaches a variety of courses in Information Systems, Statistics and Operations Management, both at the undergraduate and graduate levels. His primary research interests are in heuristics and metaheuristics for various optimization problems. E-mail: agarwala@sar.usf.edu (Corresponding author)

Selcuk Erenguc is a Full Professor and the Senior Associate Dean at the Warrington College of Business Administration at the University of Florida. He has a DBA in Quantitative Business Analysis from Indiana University (1980) and an MBA from American University of Beirut (1974). His primary research interests are in manufacturing planning and control, project planning and scheduling and supply chain management. E-mail: Selcuk.erenguc@warrington.ufl.edu 


\section{REFERENCES}

1. Agarwal, A., Colak, S. and Eryarsoy, E. (2006). Improvement Heuristic for the Flow-Shop Scheduling Problem: An Adaptive-Learning Approach. European Journal of Operational Research. 169(3): 801-815.

2. Alcaraz, J., Maroto, C. and Ruiz, R. (2003). Solving the Multi-Mode Resource-Constrained Project Scheduling Problem with genetic algorithms. Journal of the Operational Research Society. 54(6): 614-626.

3. Barrios, A., Ballestin, F. and Valls, V. (2011). A double genetic algorithm for the mrcpsp/max. Computers \& Operations Research. 38(1): 33-43.

4. Boctor, F.F. (1993). Heuristics for scheduling projects with resource restrictions and several resourceduration modes. International Journal of Production Research. 31(11): 2547-2558.

5. Boctor, F.F. (1996a). A new and efficient heuristic for scheduling projects with resource restrictions and multiple execution modes. European Journal of Operational Research, 90(2): 349-361.

6. Boctor, F.F. (1996b). Resource Constrained Project Scheduling by simulated Annealing. International Journal of Production Research. 34(8): 2335-2351.

7. Boulemein, K. and Lecocq, H. (2003). A new efficient simulated annealing algorithm for the resourceconstrained project scheduling problem and its multiple-mode version. European Journal of Operational Research. 149(2): 268-281.

8. Demeulemeester, E. and Herroelen, W. (1992). A branch-and-bound procedure for the multiple resource constrained project scheduling problem. Management Science. 38(12): 1803-1818.

9. Drexl, A. and Grunewald, J. (1993). Nonpreemptive multi-mode resource-constrained project scheduling. IIE Transactions. 25(5): 74-81.

10. Elmaghraby, S.E. (1977). Activity Networks: Project Planning and Control by Network Models. Wiley, New York, 1977.

11. Hartmann, S. and Drexl, A. (1998). Project scheduling with multiple modes: a comparison of exact algorithms. Networks. 32(4): 283-297.

12. Hartmann, S. (2001). Project scheduling with multiple modes: a genetic algorithm. Annals of Operational Research. 102(1): 111-135.

13. Jozefowska, J., Mika, M., Rozycki, R., Waligora, G. and Weglarz, J. (2001). Simulated annealing for multi-mode resource-constrained project scheduling. Annals of Operational Research, 102(1): 137-155.

14. Kolisch, R., (1996). Serial and parallel resource-constrained project scheduling methods revisited. European Journal of Operational Research. 90(2): 320-333.

15. Kolisch, R. and Drexl. A. (1997). Local search for non-preemptive multi-mode resource-constrained project scheduling. IIE Transactions. 29(11): 987-999.

16. Kolisch, R. and Sprecher, A. (1997). PSPLIB --- a project scheduling problem library. European Journal of Operational Research. 96, 205-216.

17. Lova, A., Tormos, P., Cervantes, M, and Barber, F. (2009) An efficient hybrid genetic algorithm for scheduling projects with resource constraints and multiple execution modes. International Journal of Production Economics. 117(2): 302-316.

18. Mori, M. and Tseng, C.C. (1997). A genetic algorithm for multi-mode resource constrained project scheduling problem. European Journal of Operational Research. 100(1): 134-141.

19. Nonobe K. and Ibaraki, T. (2002) Formulation and tabu search algorithm for the resource constrained project scheduling problem. Essays and Surveys in Metaheuristics. Eds. Ribeiro and P. Hansen, Kluwer Academic Publishers.

20. Ozdamar, L. (1999). A genetic algorithm approach to a general category project scheduling problem. IEEE Transactions on Systems, Man, and Cybernetics Part C. 29(1): 44-59.

21. Ozdamar, L. and Ulusoy. G. (1994). A local constraint based analysis approach to project scheduling under general resource constraints. European Journal of Operational Research. 79(2): 287-298.

22. Patterson, J.H., Slowinski, R., Talbot, F.B. and Weglarz, J. (1989). An algorithm for a general class of precedence and resource constrained scheduling problem. Advances in Project Scheduling. Eds. Jan Weglarz and Joanna Jozefowska. Elsevier: Amsterdam. 3-28.

23. Patterson, J.H., Talbot, F.B., Slowinski, R. and Weglarz, J. (1990). Computational experience with a backtracking algorithm for solving a general class of precedence and resource-constrained project scheduling problem. European Journal of Operational Research. 165(2): 375-386. 
24. Slowinski R., Soniewicki, B. and Weglarz, J. (1994). DSS for multiobjective project scheduling. European Journal of Operational Research. 79(2): 220-229.

25. Sprecher, A. and Drexl, A. (1998). Multi-mode resource-constrained project scheduling by a simple, general and powerful sequencing algorithm. European Journal of Operational Research. 107(2): 431-450.

26. Sprecher, A., Hartmann, S. and Drexl, A. (1997). An exact algorithm for project scheduling with multiple modes. OR Spektrum. 19, 195-203.

27. Talbot, F.B. (1982). Resource-constrained project scheduling with time-resource trade-offs: the nonpreemptive case. Management Science. 28(10): 1197-1210.

28. Valls, V., Ballestin, F. and Quintanilla, S. (2005). Justification and RCPSP: A technique that pays. European Journal of Operational Research. 165(2): 375-386. 


\section{NOTES}

